\title{
Effects of different regimens to lower blood pressure on major cardiovascular events in older and younger adults: meta-analysis of randomised trials
}

\author{
Blood Pressure Lowering Treatment Trialists' Collaboration
}

Correspondence to: F Turnbull, Blood Pressure Lowering Treatment Trialists' Collaboration, George Institute for International Health, University of Sydney, PO Box M201, Sydney, NSW 2050 Australia fturnbull@george.org.au

doi:10.1136/bmj.39548.738368.BE

\section{ABSTRACT}

Objective To quantify the relative risk reductions achieved with different regimens to lower blood pressure in younger and older adults.

Design Meta-analyses and meta-regression analyses used to compare the effects on the primary outcome between two age groups ( $<65 v \geq 65$ years). Evidence for an interaction between age and the effects of treatment sought by fitting age as a continuous variable and estimating overall effects across trials.

Main outcome measures Primary outcome: total major cardiovascular events.

Results 31 trials, with 190606 participants, were included. The meta-analyses showed no clear difference between age groups in the effects of lowering blood pressure or any difference between the effects of the drug classes on major cardiovascular events (all $P \geq 0.24$ ). Neither was there any significant interaction between age and treatment when age was fitted as a continuous variable (all P>0.09). The meta-regressions also showed no difference in effects between the two age groups for the outcome of major cardiovascular events ( $\$ 65 v \geq 65$; $\mathrm{P}=0.38$ ).

Conclusions Reduction of blood pressure produces benefits in younger ( 165 years) and older ( $\geq 65$ years) adults, with no strong evidence that protection against major vascular events afforded by different drug classes varies substantially with age.

\section{INTRODUCTION}

Observational studies have shown that blood pressure levels are strongly and directly related to the relative risks of stroke and heart disease ${ }^{1}$ but that the strength of the association declines with increasing age. ${ }^{2} \mathrm{~A}$ recent large overview found that for each $20 \mathrm{~mm} \mathrm{Hg}$ lower usual systolic blood pressure, the risk of stroke was $33 \%$ lower in those aged $80-89$ but $62 \%$ lower in those aged 50-59. ${ }^{3}$ While many trials with broad entry criteria for age have investigated the effects of lowering blood pressure on major vascular events, consistently larger reductions in relative risk have not been reported for younger participants. This might reflect true comparability of the effects of reducing blood pressure in older and younger people but might also be a consequence of the limited power of the individual trials to detect differences in effectiveness between age groups. Likewise, there is a paucity of evidence about the effects of different drug classes in older compared with younger patients. Some guidelines advocate the selective use of particular drug regimens based on patients' age, ${ }^{45}$ though systematic reviews quantifying the comparative effects of regimens on major vascular outcomes have not been done.

With the global population rapidly ageing ${ }^{6}$ and guidelines recommending treatment to lower blood pressure for an increasing proportion of the elderly population, we need clear evidence about the effects of such treatments in older compared with younger adults. The Blood Pressure Lowering Treatment Trialists' Collaboration was established to perform a prespecified series of overviews of trials investigating the effects of drugs to lower blood pressure on cardiovascular mortality and morbidity, including assessments of the comparative effects of regimens between major subgroups of patients. We compared the proportionate risk reductions achieved with different classes of drugs in younger and older adults.

\section{METHODS}

Trials included

Trials were eligible for inclusion if they randomised patients between a drug to lower blood pressure and control (placebo or less intensive blood pressure treatment) or randomised patients between regimens based on different classes of drug to lower blood pressure. Trials had to have a minimum of 1000 patient years of planned follow-up in each randomised group and must not have presented or published their main results before we finalised our protocol in July $1995 .^{7}$ We included in our analyses trials for which data had been obtained by September 2006. When a trial included more than two treatment arms, we calculated estimates of effect for all possible comparisons except when early termination of one arm made such estimates impossible. ${ }^{8}$ Data were accepted as data on individual patients (25 studies) or as tabular data by using prespecified categories (six studies). The data requested included participants' characteristics 


$\begin{array}{ll}\text { No of events/patients } & \begin{array}{c}\text { Difference in } \\ \text { SBP/DBP } \\ \text { Active Control }\end{array}\end{array}$

Risk ratio (95\% Cl)
Risk ratio P for (95\% Cl) homogeneity
Angiotensin converting enzyme inhibitor $v$ placebo

Age $<65813 / 9514 \quad 1087 / 9640 \quad-4.6 /-2.1$ Age $\$ 65$ 1251/8005 1490/7918 $\quad-4.2 /-2.0$
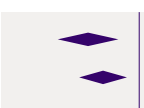

0.76 (0.66 to 0.88$)$ 0.83 (0.74 to 0.94$)$

Calcium antagonist $\boldsymbol{v}$ placebo

Age $<65 \quad 43 / 1310 \quad 49 / 1287$

Age $\$ 65 \quad 130 / 2220 \quad 170 / 2134$

$-7.2 /-2.9$

$-9.3 /-3.8$

More $\boldsymbol{v}$ less intensive blood pressure

lowering regimen

Age $<65$ 212/5024 365/9360 -3.9/-3.6

Age $\geqslant 65$ 156/2251 260/4198 $\quad-3.3 /-3.3$

0.5

Favours

active

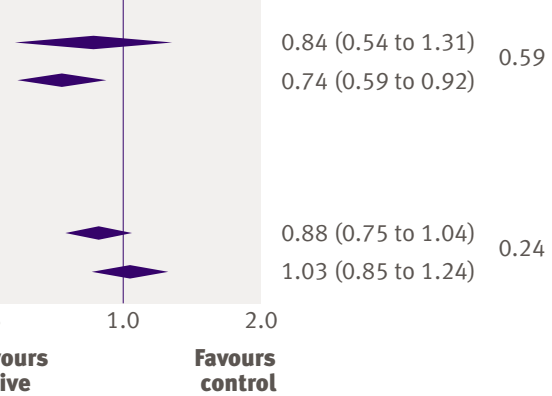

Fig 1 | Comparison of blood pressure lowering regimens against placebo or less intensive control. SBP/DBP difference=overall difference in mean blood pressure during follow-up between treatment groups (actively treated group versus control group), calculated by weighting difference observed in each contributing trial by number of individuals in trial. Negative blood pressure values indicate lower mean follow-up blood pressure in first listed than in second listed groups

recorded at screening or randomisation, selected measurements made during follow-up, and details of the occurrence of all outcomes during the scheduled follow-up period.

\section{Age groups}

The age groups predefined in the original overview protocol were $<65$ and $\geq 65$ years at the time of entry into the trial, henceforth referred to as "younger" and "older" adults. These cut offs were chosen because most participating trials used the same categories in their own subgroup analyses.

\section{Outcomes}

Our primary outcome was total major cardiovascular events, comprising stroke (non-fatal stroke or death from cerebrovascular disease), coronary heart disease (non-fatal myocardial infarction or death from coronary heart disease including sudden death) and heart failure (causing death or resulting in admission to hospital). Secondary outcomes were stroke, coronary heart disease, heart failure, cardiovascular death, and total mortality. All outcomes were prespecified in the original study protocol. ${ }^{7}$

\section{Comparisons}

The seven comparisons of treatment were those reported in the second main cycle of overviews ${ }^{9}:(a)$ angiotensin converting enzyme inhibitor versus placebo, (b) calcium antagonist versus placebo, $(c)$ more intensive versus less intensive regimens to lower blood pressure, $(d)$ angiotensin receptor blocker versus control regimen, $(e)$ angiotensin converting enzyme inhibitor versus diuretics $/ \beta$ blockers, $(f)$ calcium antagonist versus diuretics/ $\beta$ blockers, and $(g)$ angiotensin converting enzyme inhibitor versus calcium antagonists. Additional comparisons examined the separate effects of diuretics and $\beta$ blockers in different age groups as some recent guidelines have made specific recommendations about the use of these treatments in older and younger adults. ${ }^{45}$ These additional comparisons were $(a)$ angiotensin converting enzyme inhibitor or calcium antagonist versus $\beta$ blockers and $(b)$ angiotensin converting enzyme inhibitor or calcium antagonist versus diuretics.

\section{Statistical analyses}

We calculated the reduction in blood pressure in each trial arm as the mean of the differences between each participant's mean blood pressure during follow-up and their blood pressure at baseline. We then calculated the mean difference in blood pressure between randomised groups by subtracting the values for the arms compared. Mean levels of baseline characteristics and the mean difference in blood pressure between randomised groups were calculated separately for younger and older adults for each trial Overall estimates were obtained by weighting the estimates from each individual study in proportion to the number of older and younger adults in that study.

We performed three sets of analyses to explore the impact of age on the proportional risk reduction achieved with lowering blood pressure.

Firstly, we carried out meta-analyses of subgroups of participants defined according to age. For each trial and each outcome we calculated the relative risk and its variance separately for each of the two age groups according to the principle of intention to treat. Each participant could contribute only the first event in any category to the calculation for each outcome but might contribute an event to analyses of several outcomes. Overall estimates of effect and 95\% confidence intervals were calculated separately for each age group by using a random effects model and inverse variance weighting (that is, weighting by the precision of the estimate for each age group in each trial). The "meta" routine in STATA (release 9.0; Stata Corporation, College Station, TX, USA) evaluated effects of randomised treatments. Consistency of treatment effects across the age groups was tested with $\chi^{2}$ tests of homogeneity.

Secondly, we investigated interactions between treatment to lower blood pressure and age taken as a continuous variable by fitting Cox's regression models including treatment, continuous age, and their interaction. The regression (" $\beta$ ") coefficient for the latter term estimates the log ratio of relative risks, comparing the treatments, where each relative risk is the effect of a unit (here taken as 10 years) increase in age with one of the two treatments. Twenty four trials contributing data on individual participants were included in these analyses, with no comparisons being made for regimens based on angiotensin receptor blockers because data were available from only one trial. We pooled the log ratios of relative risks using inverse variance weighted random effects meta-analysis. The pooled 


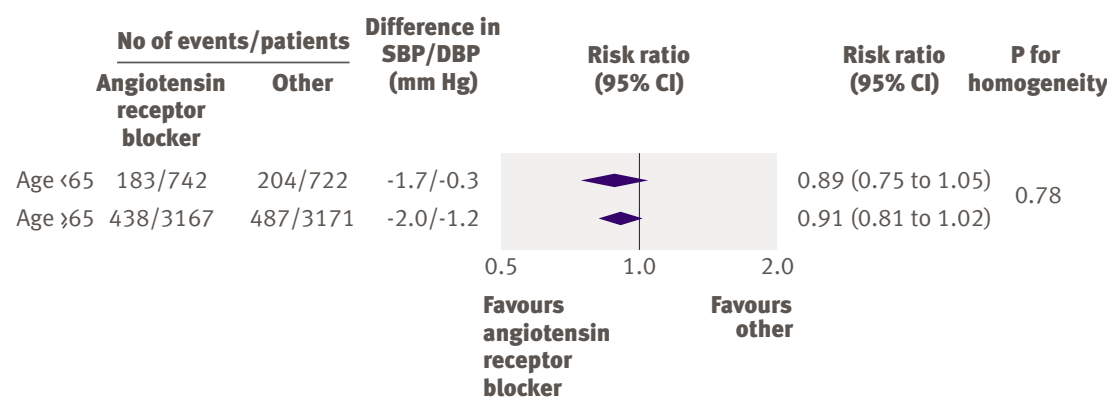

Fig 2 | Comparison of angiotensin receptor blocker based regimens with control regimens. SBP/ DBP difference $=$ overall difference in mean blood pressure during follow-up between treatment groups (angiotensin receptor blocker treated group versus control group), calculated by weighting difference observed in each contributing trial by number of individuals in trial. Negative blood pressure values indicate lower mean follow-up blood pressure in first listed than in second listed groups

summary was exponentiated to arrive at the overall estimated ratio of relative risks for a 10 year difference in age for each outcome.

Thirdly, we carried out meta-regression analyses to explore the association between the difference in systolic blood pressure at follow-up between randomised groups and the $\log$ relative risk for cardiovascular events in each age group. This was investigated across trials with random effects metaregression models with inverse variance weighting. ${ }^{10}$ Analyses were carried out using the metareg routine in STATA. We fitted separate regression lines for each age group and compared the slopes of these lines to test for a differential effect of reduction in blood pressure on risk reduction between age groups for each outcome. The model included an interaction term between age group and systolic blood pressure. ${ }^{10}$ Assumptions of linear associations between differences in blood pressure at follow-up and log relative risks were tested with standard graphical methods. As trial participants could contribute only once to a given meta-regression analysis, for factorial trials that included randomisation to different intensities of blood pressure lowering and randomisation to different drug treatments ${ }^{\mathrm{w1}-\mathrm{w} 5}$ we included only the results of the randomisation to different intensities of blood pressure lowering. For similar reasons, trials with randomisation to three treatment arms ${ }^{\mathrm{w6}-\mathrm{w} 8}$ contributed results from only two of the possible three treatment comparisons, with the control arm participants divided between the two comparisons. We carried out sensitivity analyses to examine the impact of removing the three trials that contributed participants' data exclusively to older age groups $(\geq 65 \text { years })^{\mathrm{w} 7 \mathrm{w} 9 \mathrm{w} 10}$ and the impact of removing the six trials for which data on individual participants were not available. ${ }^{\mathrm{w} 1 \mathrm{w} 9 \mathrm{w} 11-\mathrm{w} 14}$ In every analysis $\mathrm{P}<0.05$ indicated that a result was possibly not due to chance, although each case required careful interpretation, given the large numbers of comparisons.

\section{RESULTS}

Characteristics of trials and patients included

Of the 37 eligible trials, ${ }^{\text {w1-w38 }}$ we included 31 (190606 individuals) in these analyses (see table A on bmj.com). For the six remaining trials ${ }^{\text {w33-w38 }}$ we could not extract data according to criteria specified in the original study protocol. $^{7}$ There were 96466 individuals aged $<65$ and 94140 aged $\geq 65$ at baseline who contributed to the primary analyses (table 1). The mean age in the two groups was 57 and 72 and the proportion of men was $58 \%$ and 51\%, respectively. Mean baseline blood pressure was higher in the older age groups, as was the proportion of primary outcome events that comprised stroke (table 2).

\section{Meta-analyses of effects of treatments in different age groups}

For the primary outcome, total major cardiovascular events, in the trials that examined blood pressure lowering regimens compared with placebo or less active control, there was no evidence of any difference in reductions in relative risk in different age groups (all $\mathrm{P}>0.2$ for heterogeneity) (fig 1). Likewise, in the overviews of trials comparing blood pressure lowering regimens based on different drug classes there was no difference in the proportional reductions in total major cardiovascular events observed between age groups for any comparison (all $\mathrm{P}>0.3$ for heterogeneity) (figs 2 and 3). While there was some variation in the reductions in blood pressure with the randomised treatments between age groups, there was no systematic pattern. Among the 35 comparisons between age groups made for the

Table 1 | Mean baseline characteristics and differences in blood pressure at follow-up between randomised groups in subgroups of younger and older adults

\begin{tabular}{|c|c|c|c|c|c|c|c|c|c|c|}
\hline \multirow[b]{2}{*}{$\begin{array}{l}\text { Treatment } \\
\text { comparison }\end{array}$} & \multicolumn{5}{|c|}{ Age $<65(n=96466)$} & \multicolumn{5}{|c|}{ Age $\geq 65(n=94140)$} \\
\hline & Total No & $\begin{array}{c}\text { Age } \\
\text { (years) }\end{array}$ & $\begin{array}{l}\text { Baseline SBP/ } \\
\text { DBP }(\mathrm{mm} \mathrm{Hg})\end{array}$ & $\begin{array}{l}\text { Difference in SBP/DBP } \\
(\mathrm{mm} \mathrm{Hg})\end{array}$ & $\%$ Men & Total No & $\begin{array}{c}\text { Age } \\
\text { (years) }\end{array}$ & $\begin{array}{l}\text { Baseline SBP/ } \\
\text { DBP }(\mathrm{mm} \mathrm{Hg})\end{array}$ & $\begin{array}{l}\text { Difference in SBP/DBP } \\
(\mathrm{mm} \mathrm{Hg})\end{array}$ & $\%$ Men \\
\hline ACE-I $v$ placebo & 19154 & 57 & $137 / 82$ & $-4.6 /-2.1$ & 79 & 15923 & 70 & $143 / 80$ & $-4.2 /-2.0$ & 72 \\
\hline CA vplacebo & 2597 & 58 & $149 / 83$ & $-7.2 /-2.9$ & 60 & 4354 & 72 & $168 / 84$ & $-9.3 /-3.8$ & 37 \\
\hline More $v$ less* & 15335 & 57 & $165 / 104$ & $-4.3 /-3.5$ & 57 & 6647 & 70 & $173 / 104$ & $-3.5 /-3.4$ & 47 \\
\hline ARB $v$ other & 1464 & 56 & $151 / 85$ & $-1.7 /-0.3$ & 64 & 6338 & 75 & $163 / 89$ & $-2.0 /-1.2$ & 39 \\
\hline $\mathrm{ACE}-\mathrm{I} v \mathrm{D} / \mathrm{BB}$ & 22088 & 55 & $153 / 93$ & $1.3 / 0.2$ & 54 & 25342 & 73 & $161 / 88$ & $2.0 / 0.5$ & 49 \\
\hline $\mathrm{CA} v \mathrm{D} / \mathrm{BB}$ & 43594 & 58 & $154 / 93$ & $1.1 /-0.2$ & 50 & 46185 & 72 & $157 / 87$ & $0.5 /-0.4$ & 46 \\
\hline ACE-I $v$ CA & 10049 & 59 & $145 / 87$ & $0.9 / 0.6$ & 55 & 16310 & 73 & $160 / 87$ & $1.0 / 1.0$ & 49 \\
\hline
\end{tabular}

$\mathrm{SBP} / \mathrm{DBP}=$ systolic/diastolic blood pressure; $\mathrm{ACE}-\mathrm{I}=$ angiotensin converting enzyme inhibitor; $\mathrm{CA}=$ calcium antagonist; $\mathrm{ARB}=$ angiotensin receptor blocker; $\mathrm{D} / \mathrm{BB}=\mathrm{diuretic}$ or $\beta$ blocker. *More $v$ less intensive blood pressure lowering regimen. 


$\frac{\text { No of events/patients }}{\text { 1st listed 2nd listed }} \begin{gathered}\begin{array}{c}\text { Difference in } \\ \text { SBP/DBP } \\ \text { (mm Hg) }\end{array} \\ \text { (mm }\end{gathered}$

\section{Risk ratio}

(95\% Cl)
Risk ratio $\quad P$ for (95\% Cl) homogeneity
Angiotensin converting enzyme inhibitor $v$ diuretic or $\boldsymbol{\beta}$ blocker

Age $<65 \quad 819 / 9448 \quad 1066 / 12012 \quad 1.3 / 0.1$

Age 365 1795/10 783 2525/14 $429 \quad 2.0 / 0.5$

Calcium antagonist $\boldsymbol{v}$ diuretic or $\boldsymbol{\beta}$ blocker

Age $<65$ 1165/20 358 1430/23 236 1.1/-0.2

Age \$65 2653/21 204 3363/24 981 0.5/-0.4

Angiotensin converting enzyme inhibitor $v$ calcium antagonist

Age $<65 \quad 548 / 5130 \quad 568 / 4919 \quad 0.9 / 0.6$

Age $\$ 65 \quad 1583 / 8170 \quad 1608 / 8140 \quad 1.0 / 1.0$

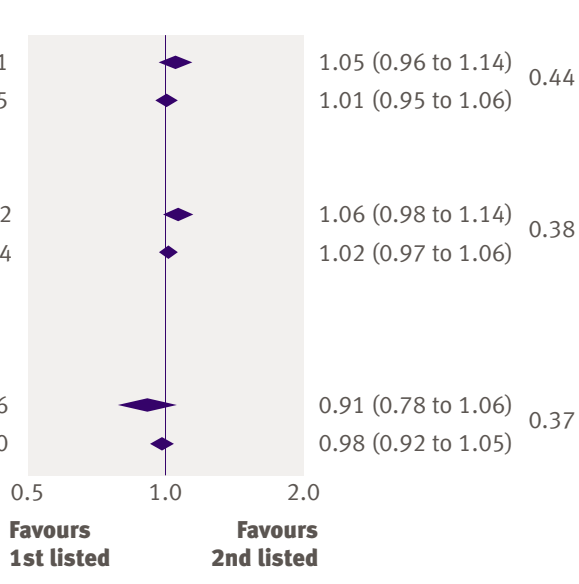

Fig 3 Blood pressure lowering regimens based on different drug classes for the outcome total major cardiovascular events and age groups $<65$ versus $\geq 65$. SBP/DBP difference $=$ overall difference in mean blood pressure during follow-up between treatment groups (group assigned first listed treatment versus group assigned second listed treatment), calculated by weighting difference observed in each contributing trial by number of individuals in trial. Negative blood pressure values indicate lower mean follow-up blood pressure in first listed than in second listed groups among the 30 analyses, and this is most likely to have arisen by chance (www.thegeorgeinstitute.org/bplttc).

Meta-regressions of effects of blood pressure lowering in different age groups

There was no difference in the risk reduction achieved per unit reduction in blood pressure for individuals aged $<65$ compared with $\geq 65$ for the primary outcome of total major cardiovascular events $(\mathrm{P}=0.38$ ) (fig 6 ) nor for any of the secondary outcomes (all $\mathrm{P} \geq 0.18$ ) (available from author (www.thegeorgeinstitute.org/bplttc).

\section{DISCUSSION}

\section{Principal findings}

These analyses provide strong support for the use of drugs to lower blood pressure in older and younger adults, with no strong evidence for the selective use of specific classes of drug according to age. While some current management guidelines advocate the use of particular types of drug according to age on the basis of possible differences in effects on major cardiovascular events, ${ }^{4511}$ factors such as tolerability and cost are probably reasonable bases for choice of drug so long as effective blood pressure reduction is achieved. ${ }^{12-14}$ In particular, for these age groups there was no evidence of differences between the effects of $\beta$ blockers and other classes of drugs in older compared with younger adults for any outcome studied, and the same was true for all other drug comparisons.

\section{Findings in context of observational studies}

We might expect variation in the effect of lowering blood pressure because observational data have shown less strong proportional associations of blood pressure levels with risk in older compared with younger adults. ${ }^{3}$ The analyses prespecified in the original overview protocol ${ }^{7}$ identified no attenuation of risk reduction with age but were not especially well powered to detect such effects. Each of the subsidiary analyses provided better statistical power, and there were some comparisons that showed evidence of different effects of blood pressure lowering between age groups or interactions between age and particular drug regimens. It is important, however, that the "statistically significant" subsidiary analyses are interpreted in light of their post hoc nature, the multiple comparisons made, and, in the case of the meta-regressions, the non-randomised nature of the evaluations. So, our results do not completely exclude the possibility of differences in the proportional effects of blood pressure lowering regimens

Effects of age on blood pressure lowering with age fitted as continuous variable

We found no evidence of an interaction between age and the effects of treatment on the primary outcome of major cardiovascular events for any blood pressure lowering treatments compared with control (all $\mathrm{P}>0.09$ ) (fig 5). The same was true for the comparisons of different active agents (all $\mathrm{P}>0.2$ ). For the secondary outcomes there was one significant interaction $(\mathrm{P}=0.02)$
Table 2 | Numbers (percentages) of individuals with stroke, coronary heart disease, and heart failure by age

\begin{tabular}{lcccc} 
Age (years) & No & Stroke & $\begin{array}{c}\text { Coronary heart } \\
\text { disease }\end{array}$ & Heart failure \\
Age $<65$ & 96466 & $2096(2.2)$ & $3624(3.8)$ & $1030(1.1)$ \\
\hline Age $\geq 65$ & 94140 & $4490(4.8)$ & $5776(6.1)$ & $2460(2.6)$ \\
\hline
\end{tabular}




$$
\frac{\text { No of events/patients }}{\text { 1st listed 2nd listed }} \begin{gathered}
\begin{array}{c}
\text { Difference in } \\
\text { SBP/DBP } \\
\text { (mm Hg) }
\end{array} \\
\text { (mm }
\end{gathered}
$$
$(95 \% \mathrm{Cl})$ homogeneity

Angiotensin converting enzyme inhibitor or calcium antagonist $\boldsymbol{v} \boldsymbol{\beta}$ blocker

Age $<65 \quad 297 / 7013 \quad 308 / 7058 \quad 0.2 / 0.1$

Age $\geqslant 65 \quad 519 / 6484 \quad 569 / 6648 \quad-0.8 /-0.2$

Angiotensin converting enzyme inhibitor or calcium antagonist $\boldsymbol{v}$ diuretic

Age $<65 \quad 923 / 8447 \quad 1455 / 13683 \quad 1.9 / 0.0$

Age $\geqslant 65$ 2412/13 $613 \quad 3786 / 20783 \quad 1.6 / 0.0$

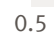

Favours

1st listed

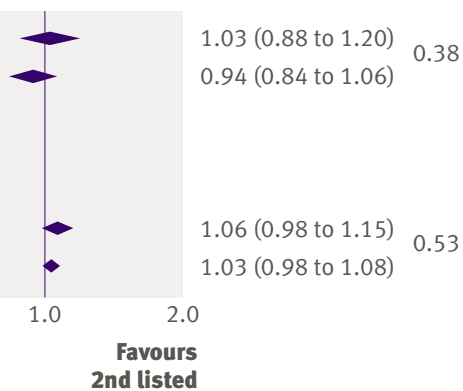

Fig 4 Regimens based on diuretics and $\beta$ blockers versus other active agents for total major cardiovascular events according to age. SBP/DBP difference=overall difference in mean blood pressure during follow-up between group assigned first listed treatment versus group assigned diuretic or $\beta$ blocker, calculated by weighting difference observed in each contributing trial by number of individuals in trial. Negative blood pressure values indicate lower mean follow-up blood pressure in first listed than in second listed groups

between age groups but they do suggest that any such differences are likely to be small.

\section{Clinical implications}

As the magnitude of the proportional risk reduction achieved with blood pressure lowering does not seem to decline with age, our findings provide strong support for the use of blood pressure lowering in elderly people. Among the older age group there was, in almost every analysis and for almost every outcome, an estimate of effect suggesting benefit from blood pressure lowering, and in no case was there evidence of harm. The much greater absolute risk in elderly people means that even if proportional reductions were attenuated in this group, the protection afforded would still translate into large numbers of serious vascular events prevented. These data also provide considerable reassurance that current approaches to the use of blood pressure lowering treatments based on absolute risk, that assume constant proportional risk reductions across age groups, ${ }^{511-14}$ are an appropriate means of quantifying the likely absolute benefit to be gained from lowering blood pressure. ${ }^{3}$

\section{Strengths and weaknesses}

Our analyses included thousands of major cardiovascular events and provided reasonably precise estimates of the effects of the different regimens in older and younger adults for most outcomes. They are, however, subject to several limitations and need to be interpreted with these in mind. Firstly, the difference in mean age between the older and younger participants was not large - only about 15 years. The observational data suggest that proportional differences in risk reduction would be modest for age differences of this magnitude, and it is possible that these overviews could have failed to detect real differences in the effectiveness of blood pressure lowering between age groups. That said, the analyses with age fitted as a continuous variable had much better statistical power to detect interactions between age and treatment to lower blood pressure and provide reassurance that moderate or large effects have not been missed. Secondly, because most patients in the trials fell within a fairly limited age range, the analyses were unable to define the effects of blood pressure lowering agents in very elderly people. Although particular concerns about the safety and efficacy of treatment to lower blood pressure in this group have previously been raised, ${ }^{15}$ recent results from the HYVET study ${ }^{16}$ have largely addressed uncertainty around the benefits of lowering blood pressure in patients aged $\geq 80$. In the same way that the limited age range of patients in our analyses could not define the effects in the very elderly, postulated differences between the effects of drug regimens in younger age groups ${ }^{17}$ cannot be excluded. Thirdly, although there was reasonable comparability in the baseline characteristics of younger and older patients it is possible that different levels of baseline blood pressure, the proportion of men, and possibly other comorbidities might have had an effect on the potential to detect differences between the age groups. Fourthly, the overviews defined only the short to medium term effects of the regimens studied and cannot exclude the evolution of differences between the effects in each age group in the longer term. Fifthly, the ability of these analyses to detect differences between regimens would have been diminished by incomplete adherence to

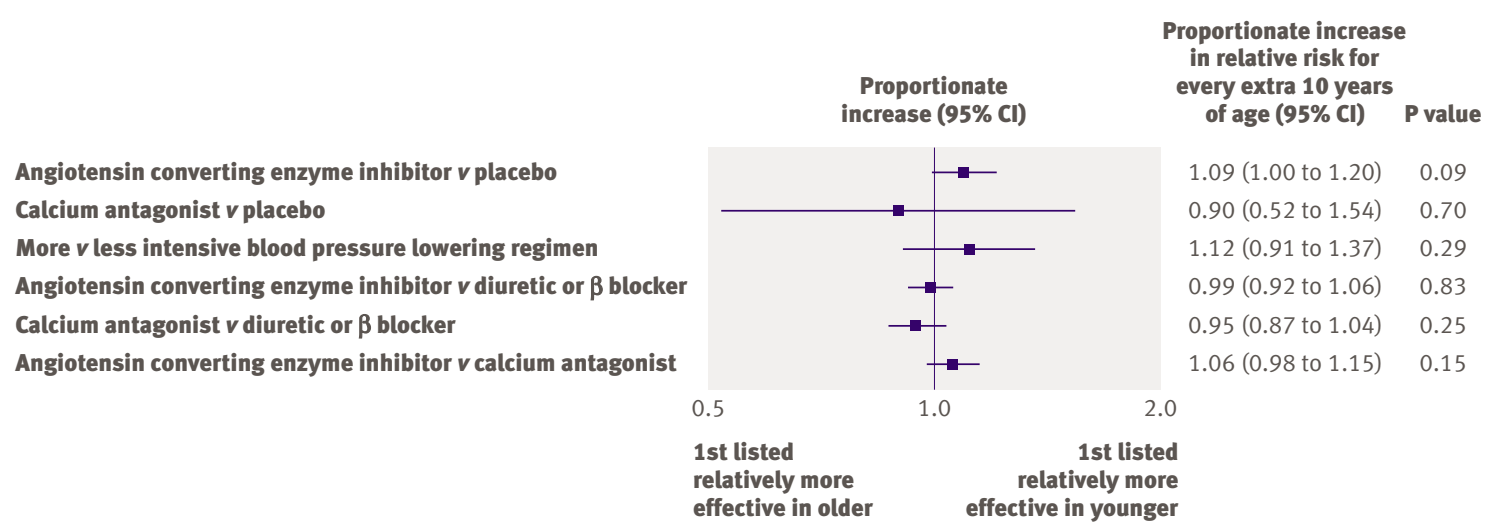

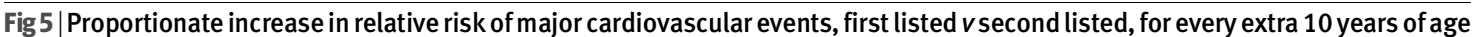


Reduction in risk for each $5 \mathrm{~mm} \mathrm{Hg}$ reduction in systolic blood pressure: - - Age $<65: 11.9 \%$ (5.3\% to $18.0 \%)$

- Age $\geqslant 65: 9.1 \%$ (3.6\% to $14.3 \%$ ) $\mathrm{P}$ for heterogeneity of slopes $=0.38$

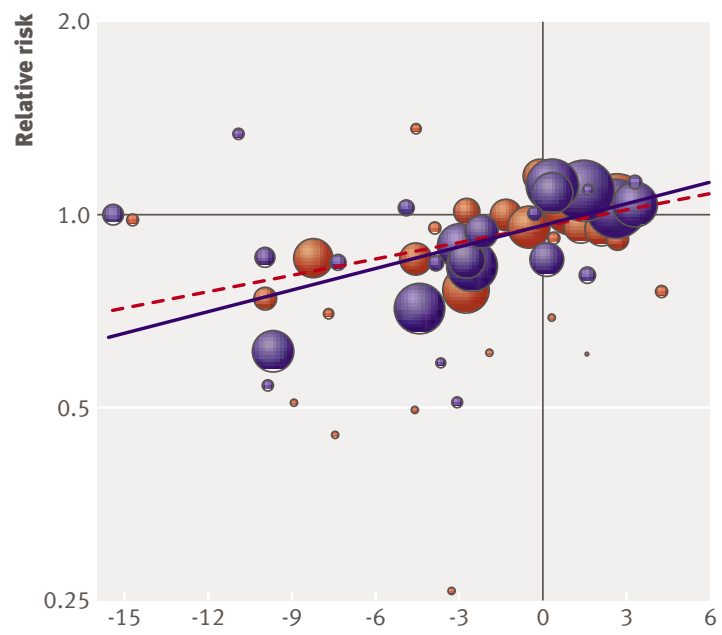

Difference in reduction in systolic blood pressure $(\mathrm{mm} \mathrm{Hg})$

Fig 6 | Associations of reduction in blood pressure with risk reduction for total major cardiovascular events for adults aged $<65$ and $\geq 65$. Area of each circle is proportional to inverse variance of log odds ratio. Fitted lines represent summary metaregressions for total major cardiovascular events

randomised treatments and the extensive use of add-on therapies. Similarly, the a priori definition of a "conventional group" that combines two different drug classes (diuretics and $\beta$ blockers) represents an additional challenge, although subsidiary analyses were not able to detect differences when $\beta$ blockers and diuretics were considered separately. Sixthly, data defined by the prespecified criteria were not available for all eligible trials, but limited subsidiary analyses including published data from those studies did not change the overall study conclusions.

Finally, the primary analyses were based on the composite outcome of major cardiovascular events, and this outcome might underestimate any real difference in the proportional effects of blood pressure lowering between age groups. The reason for this is that

\section{WHAT IS ALREADY KNOWN ON THIS TOPIC}

In observational studies the proportional reductions in the risks of vascular disease associated with lower blood pressure levels decline with increasing age, suggesting that the relative effects of blood pressure lowering drugs might be smaller among elderly people

Some blood pressure management guidelines recommend specific classes of blood pressure lowering treatment for particular age groups

\section{WHAT THIS STUDY ADDS}

Blood pressure reduction produces similar proportional reductions in the risks of vascular events in younger ( 165 years) and older ( $\geq 65$ years) adults

The absolute benefits of treatment are likely to be particularly large among older individuals because of their higher average risk

There was no clear evidence to support recommendations for particular drug classes in older or younger adults the composition of major cardiovascular events varies between age groups, with the proportion of strokes, which are more strongly affected by lowering blood pressure, being larger in the older age groups. This would tend to inflate the magnitude of the proportional reduction achieved for major cardiovascular events in the older compared with the younger group. The secondary analyses of the separate major cause-specific outcomes do not, however, suggest that this has been a major cause of confounding.

\section{Conclusions}

Our results confirm the benefits of effective control of blood pressure in older and younger adults. They also provide substantial reassurance that, within the age range studied, the benefits of regimens to lower blood pressure based on different drug classes are largely comparable across age groups, although there is a relative paucity of data for those under 50 and over 80 . None the less, these findings should greatly simplify decision making for millions of clinicians around the world.

Members of the Blood Pressure Lowering Treatment Trialists'

Collaboration: L Agodoa (National Institute of Diabetes and Digestive and Kidney Diseases, National Institutes of Health, Bethesda, Maryland, US) C Anderson, J Chalmers, S MacMahon, B Neal (George Institute, Sydney, Australia); F W Asselbergs, W H van Gilst (University of Groningen, Groningen, Netherlands); C Baigent, R Collins (Clinical Trial Service Unit, University of Oxford); H Black (New York University School of Medicine, New York, US); B Brenner, M Pfeffer (Brigham and Women's Hospital, Boston, US); C Bulpitt, P Poole-Wilson (Imperial College, London); R Byington (Wake Forest University, Winston-Salem, US); J Cutler (Nationa Heart, Lung and Blood Institute, Bethesda, US); B Davis (University of Texas School of Public Health, Houston, US); D de Zeeuw (University Medical Center Groningen, Groningen, Netherlands); J Dens (University Hospital Gasthuisberg, Leuven, Belgium); R Estacio (University of Colorado Health Sciences Center, Denver, US); R Fagard (University of Leuven, K U Leuven, Belgium); K Fox (Royal Brompton Hospital and Imperial College, London); T Fukui (St Luke's International Hospital, Tokyo Japan); L Hansson (deceased), R Holman (Oxford Centre for Diabetes, Endocrinology and Metabolism, University of Oxford); Y Imai, T Ohkubo (Tohoku University Graduate School of Pharmaceutical Sciences and Medicine, Sendai, Japan); M Ishii (Yokohama Seamen's Insurance Hospital, Yokohama, Japan); Y Kanno, H Suzuki (Saitama Medical University, Saitama, Japan); J Kostis (UMDNJ-Robert Wood Johnson Medical School, New Brunswick, US); K Kuramoto (Tokyo Metropolitian Geriatric Hospital, Tokyo, Japan); E Lewis (Rush University Medical Center, Chicago, US); M Lièvre (Louis Pradel Hospital Université Claude Bernard-Lyon 1, Lyon, France); L H Lindholm (Department of Public Health and Clinical Medicine, Umeå University, Sweden); L Liu (Fu Wai Hospital and Cardiovascular Institute, Beijing, China); I Lubsen (SOCAR Research S.A, Nyon, Switzerland); S Lueders, J Schrader (St Josefs Hospital, Cloppenburg, Germany); E Malacco (Ospedale L. Sacco, University of Milan, Italy); G Mancia (University of Milano-Bicocca, Department of Clinical Medicine and Prevention, San Gerardo Hospital, Milan, Italy); M Matsuzaki (Yamaguchi University Hospital, Yamaguchi, Japan); S Nissen (Cleveland Clinic, Cleveland, US); T Ogihara (Osaka University Graduate School of Medicine, Osaka, Japan); C Pepine (University of Florida, Gainsville, US); B Pitt (University of Michigan School of Medicine, Ann Arbor, US); M Rahman (University Hospitals of Cleveland Case Medical Center, Cleveland, US); W Remme (Sticares Cardiovascular Research Institute, Rhoon, Netherlands); G Remuzzi, P Ruggenenti (Mario Negri Institute for Pharmacological Research and Ospedali Riuniti di Bergamo, Bergamo, Italy); A Rodgers (Clinical Trials Research Unit, University of Auckland, New Zealand); T Saruta (Keio University School of Medicine, Tokyo, Japan);, R Schrier (University of Colorado School of Medicine, Denver, US); P Sleight (University of Oxford and John Radcliffe Hospital, Oxford); J Staessen (University of Leuven, Leuven, Belgium); K Teo (McMaster University Medical Centre, Ontario, Canada); G Viberti (King's College London, Guy's Hospital, London); J Wang (Ruijin Hospital, Shanghai, China); P Whelton 
(Loyola University Medical Center, Maywood, US); L Wing (Flinders University, Adelaide, Australia); Y Yui (Kyoto University Hospital, Kyoto, Japan); S Yusuf (Hamilton General Hospital, Ontario, Canada); A Zanchetti (University of Milan and Instituto Auxologico Italiano, Milan, Italy). Collaboration Coordinating Centre (George Institute for International Health, Sydney, Australia): F Barzi, J Chalmers, S Heritier, N Li, S MacMahon, B Neal, T Ninomiya, V Perkovic, F Turnbull, M Woodward. Writing committee: F Turnbull, B Neal, T Ninomiya, C Algert, H Arima,

F Barzi, C Bulpitt, J Chalmers, R Fagard, A Gleason, S Heritier, N Li, V Perkovic, M Woodward, S MacMahon.

Contributors: $\mathrm{FT}$ is guarantor.

Funding: National Health and Medical Research Council of Australia. B Neal was supported by a fellowship awarded by the National Heart Foundation of Australia. T Ninomiya was supported by a fellowship awarded by the Banyu Life Science Foundation and by an International Society of Hypertension visiting postdoctoral fellowship awarded by the Foundation for High Blood Pressure Research Council of Australia. V Perkovic was supported by a postdoctoral fellowship awarded by the National Heart Foundation of Australia.

Competing interests: Of the members of the writing committee , FT, BN, TN, HA, CB, JC, VP, MW, and SM have received travel expenses, payment for speaking at meetings, or research funding from one or more agencies that sponsored trials included in this collaboration.

Ethical approval: Not required.

Provenance and peer review: Not commissioned; externally peer reviewed.

1 MacMahon S, Peto R, Cutler J, Collins R, Sorlie P, Neaton J, et al. Blood pressure, stroke, and coronary heart disease. Part 1. Prolonged differences in blood pressure: prospective observational studies corrected for the regression dilution bias. Lancet 1990;335:765-74

2 Prospective Studies Collaboration. Cholesterol, diastolic blood pressure, and stroke: 13,000 strokes in 450,000 people in 45 prospective cohorts. Lancet 1995;346:1647-53.

3 Prospective Studies Collaboration. Age-specific relevance of usual blood pressure to vascular mortality: a meta-analysis of individual data for one million adults in 61 prospective studies. Lancet 2002;360:1903-13.

4 Williams B, Poulter N, Brown M, Davis M, McInnes G, Potter J, et al. British Hypertension Society Guidelines for management of hypertension: report of the fourth working party of the British Hypertension Society, 2004-BHS IV. J Hum Hypertens 2004;18:139-85.
5 Canadian Hypertension Education Program. 2007 guidelines for the management of arterial hypertension. Can J Cardiol 2007;23:539-50.

6 US Census Bureau. Global population profile. Washington, DC: US Government Printing Office, 2002. (Report No. Report WP/02.)

7 World Health Organization-International Society of Hypertension Blood Pressure Lowering Treatment Trialists' Collaboration. Protocol for prospective collaborative overviews of major randomized trials of blood-pressure lowering treatments. J Hypertens 1998;16:127-37.

8 ALLHAT Officers and Coordinators for the ALLHAT Collaborative Research Group. Major cardiovascular events in hypertensive patients randomized to doxazosin vs chlorthalidone: the antihypertensive and lipid-lowering treatment to prevent heart attack trial (ALLHAT). JAMA 2000;283:1967-75.

9 Blood Pressure Lowering Treatment Trialists' Collaboration. Effects of different blood pressure lowering regimens on major cardiovascular events: Second cycle of prospectively designed overviews. Lancet 2003;362:1527-35.

10 Woodward M. Epidemiology: study design and data analysis. 2nd ed. Boca Raton: Chapman and Hall/CRC Press, 2005.

11 National Collaborating Centre for Chronic Conditions. Hypertension: management in adults in primary care: pharmacological update. London: Royal College of Physicians, 2006.

12 Chobanian A, Bakris G, Black H, Cushman W, Green L, Izzo J, et al. The seventh report of the Joint National Committee on prevention, detection, evaluation and treatment of high blood pressure. The JNC 7 report. JAMA 2003;289:2560-72.

13 Task Force for the Management of Arterial Hypertension of the European Society of Hypertension (ESH) and of the European Society of Cardiology (ESC). 2007 guidelines for the management of arterial hypertension. J Hypertens 2007;25:1105-87.

14 World Health Organization. 2003 World Health Organization (WHO)/ International Society of Hypertension (ISH) statement on management of hypertension. J Hypertens 2003;21:1983-92.

15 Gueyffier F, Bulpitt C, Boissel J, Schron E, Ekbom T, Fagard R, Casiglia E, Kerlikowske K, Coope J. Antihypertensive drugs in very old people: a subgroup meta-analysis of randomised controlled trials. Lancet 1999;353:793-96.

16 Beckett N, Peters R, Fletcher A, Staessen J, Liu L, Dumitrascu D, et al. Treatment of hypertension in patients 80 years of age or older. NEnglJ Med 2008 Mar [31 Epub ahead of print].

17 Brown M, Cruickshank J, Dominiczak A, MacGregor G, Poulter N, Russell G. Better blood pressure control: how to combine drugs.J Hum Hypertens 2003;17:81-6.

Accepted: 4 April 2008 